\title{
Soft elasticity and microstructure in smectic C elastomers
}

August 21, 2006

\author{
James Adams ${ }^{1}$, Sergio Conti ${ }^{1}$, and Antonio DeSimone ${ }^{2}$ \\ ${ }^{1}$ Fachbereich Mathematik, Universität Duisburg-Essen \\ Lotharstr. 65, 47057 Duisburg, Germany \\ 2 SISSA, International School for Advanced Studies \\ Via Beirut 2-4, 34014 Triste, Italy
}

\begin{abstract}
Smectic C elastomers are layered materials exhibiting a solidlike elastic response along the layer normal and a rubbery one in the plane. The set of strains $K_{\mathrm{C}}$ minimizing the elastic energy contains a one-parameter family of simple stretches associated with an internal degree of freedom, coming from the in-plane component of the director. We investigate soft elasticity and the corresponding microstructure by determining the quasiconvex hull of the set $K_{\mathrm{C}}$, and use this to propose experimental tests that should make the predicted soft response observable.
\end{abstract}

\section{Introduction}

Liquid crystal elastomers display a number of interesting mechanical and optical properties, with potential applications ranging from nonlinear optics to artificial muscles, due to the coupling between liquid crystal ordering transition and rubber elasticity [25]. Smectic C liquid crystals are characterized by rod-like molecules (mesogens) assembled in a layered structure, and tilted at a fixed angle with respect to the layer normal (see Figure 1). The mesogens are attached to polymer chains, which are cross-linked to obtain a rubberlike solid; the coupling to liquid crystal ordering leads to rubbery response in the tangential directions, and solid-like along the layer normal. Monodomain smectic C elastomers were recently synthesized [14] (see also discussion and references in [25, Chapter 12]); they exhibit spontaneous shear at the smectic A-smectic C phase transition [15]. Experimental interest in smectic C elastomers is also strongly motivated by the existence of a chiral ferroelectric phase.

A microscopic model based on statistical mechanics was recently derived in [2]. The model treats the polymers as Gaussian coils, with the cross-linkers 


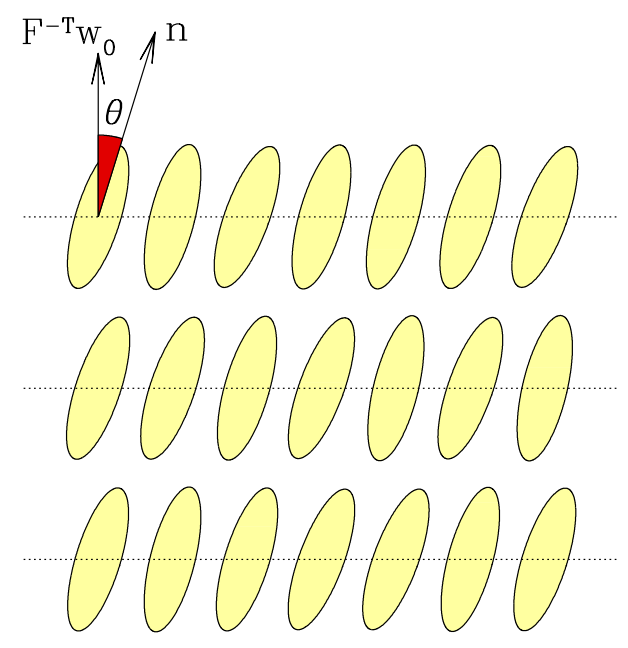

FiguRE 1: Sketch of the geometry of smectic $\mathrm{C}$ elastomers. The unit vector $n$ gives the mesogen orientation in the deformed configuration, $F$ is the deformation gradient, $F^{-T} w_{0}$ (or, equivalently, cof $F w_{0}$ ) gives the normal to the smectic layers in the deformed configuration.

constrained to lie between the smectic layers. The free energy is obtained by taking a quenched average over the distribution of the chain endpoints at cross-linking. This leads to an elastic stored energy density expressed as a function of the local deformation gradient, see below.

In [2] soft modes were predicted, i.e., a nontrivial set $K_{\mathrm{C}}$ of energyminimizing deformations was identified. Soft modes have also been predicted based on a phenomenologically derived Lagrangian elastic energy [23]. We show here that $K_{\mathrm{C}}$ is not quasiconvex, and hence that zero-energy microstructures can be formed. In particular, we find that the set of macroscopic strains which can be realized with zero energy is much larger than $K_{\mathrm{C}}$. We characterize all such zero-energy macroscopic deformations by computing the quasiconvex hull of $K_{\mathrm{C}}$. Our approach is based on the tools of the calculus of variations (see, e.g., $[3,9,17,12]$ ), which have been remarkably successful, e.g., for shape-memory alloys [5] or nematic elastomers [25, Chapter 8].

The explicit computation of quasiconvex hulls in realistic three-dimensional physical systems is far from straightforward, even more so when working with finite deformations. Examples where this has been possible are nematic elastomers [11], and (at least partially) crystalline solids undergoing a cubic-totetragonal martensitic phase transition [13]. By computing the quasiconvex hull for smectic $\mathrm{C}$ elastomers and by exploiting this result to discuss the experimental accessibility of soft deformation paths we provide a further example in which the variational approach proves successful. The application 
of these techniques to predict the result of stretching experiments on liquid crystal elastomers has been demonstrated in $[6,7,8]$.

\section{Statement of the problem and main results}

The statistical mechanical model [2] gives the elastic stored energy density

$$
W_{\mathrm{C}}(F)=\left\{\begin{array}{cl}
\frac{1}{2} \mu \min _{n \in N^{(F)}}\left[|F|^{2}+\left(\frac{1}{\alpha^{2}}-1\right)\left|F^{T} n\right|-3\right] \\
+\frac{1}{2} B\left(\frac{\left|U_{0}^{-1} w_{0}\right|}{\left|F^{-T} w_{0}\right|}-1\right)^{2} & \text { if } \operatorname{det} F=\alpha \\
+\infty & \text { else. }
\end{array}\right.
$$

Here $F \in \mathbb{R}^{3 \times 3}$ is the deformation gradient relative to a reference configuration chosen in order to fully exploit the cylindrical symmetry of the material (see Appendix), $U_{0}=\operatorname{Id}+(\alpha-1) n_{0} \otimes n_{0}$ is the deformation gradient at cross-linking, $w_{0}$ is a fixed unit vector chosen such that $U_{0}^{-1} w_{0}$ is orthogonal to the smectic layers at cross linking,

$$
N^{(F)}=\left\{n \in S^{2}: n \cdot \frac{F^{-T} w_{0}}{\left|F^{-T} w_{0}\right|}=\cos \theta\right\}
$$

and $n_{0} \in N^{\left(U_{0}\right)}$ is the orientation of the mesogens at cross-linking. The quantities $\mu>0, B>0, \alpha>1$, and $\theta \in(0, \pi / 2)$ are material parameters. This expression differs from the one given in [2] by a change of variables in the reference configuration, see Appendix. The fact that the energy $W_{\mathrm{C}}$ is only finite on matrices with fixed determinant reflects the assumption of incompressibility by which all deformations from the initial configuration are volume-preserving. The choice of the value $\alpha$ is simply dictated by notational convenience. The parameter $\alpha$ is physically related to the anisotropy of the polymer chains and hence to the amplitude of the spontaneous deformations (see Section 3); $\theta$ characterizes the angle formed by the director with the layer normal (in particular, the case of smectic A elastomers is recovered for $\theta=0)$; and $B \gg \mu$ are the elastic moduli normal and tangential to the layer normal.

The function $W_{\mathrm{C}}$ defined in (2.1) is nonnegative, its minimum is zero, and is attained by matrices in the set

$$
K_{\mathrm{C}}=S O(3)\left\{\operatorname{Id}+(\alpha-1) n \otimes n: n \cdot w_{0}=\beta\right\}=S O(3) \bigcup_{n \in N_{\mathrm{C}}} U_{n}
$$


where

$$
U_{n}=\mathrm{Id}+(\alpha-1) n \otimes n
$$

and

$$
N_{\mathrm{C}}=\left\{n: n \in S^{2}, n \cdot w_{0}=\beta\right\}
$$

(see Appendix for a proof of these simple facts). Here $\beta \in(0,1)$ is a parameter related to the cosine of the angle $\theta$ between layer normal and director $n$, see (A.4).

A function $W: \mathbb{R}^{3 \times 3} \rightarrow \mathbb{R} \cup\{\infty\}$ is said to be quasiconvex if affine boundary conditions permit affine minimizers. Precisely, one requires that for all $F \in \mathbb{R}^{3 \times 3}$, and all open sets $\Omega \subset \mathbb{R}^{3}$, the energy of the affine map $x \mapsto F x$ cannot be reduced without changing the boundary conditions, in the sense that

$$
|\Omega| W(F) \leq \int_{\Omega} W(F+\nabla y) d x
$$

for all $y \in W_{0}^{1, \infty}\left(\Omega ; \mathbb{R}^{3}\right)$ for which the integral exists. Given a compact set $K \subset \mathbb{R}^{3 \times 3}$, one defines its quasiconvex hull as the set of points which cannot be separated from $K$ by quasiconvex functions, i.e.,

$$
\begin{aligned}
& K^{\mathrm{qc}}=\left\{F \in \mathbb{R}^{3 \times 3}: W(F) \leq \inf _{F^{\prime} \in K} W\left(F^{\prime}\right)\right. \\
&\text { for all } \left.W: \mathbb{R}^{3 \times 3} \rightarrow \mathbb{R} \text { quasiconvex. }\right\} .
\end{aligned}
$$

Physically, the set $K^{\mathrm{qc}}$ consists of the macroscopic deformation gradients which can be realized with zero energy. Indeed, it can be shown that $K^{\mathrm{qc}}$ is the set of matrices $F \in \mathbb{R}^{3 \times 3}$ such that there is a bounded sequence $y_{j} \in$ $W^{1, \infty}\left(\Omega ; \mathbb{R}^{3}\right)$ such that $y_{j}(x)=F x$ on $\partial \Omega$ for all $j$, and $\operatorname{dist}\left(\nabla y_{j}, K\right) \rightarrow 0$ in measure (this is true for any open and bounded set $\Omega \subset \mathbb{R}^{3}$ with $|\partial \Omega|=0$ ). For a more detailed presentation of these concepts see, e.g., [9, 17, 12].

The following theorem gives an explicit formula for $K_{\mathrm{C}}^{\mathrm{qc}}$.

Theorem 2.1. Let $K_{\mathrm{C}}$ be as in (2.2), with $\beta \in(0,1), \alpha>1$, and $w_{0}$ a unit vector. Then,

$$
\begin{aligned}
K_{\mathrm{C}}^{\mathrm{qc}}= & \left\{F \in \mathbb{R}^{3 \times 3}: \operatorname{det} F=\alpha, \quad \lambda_{1}(F) \geq 1\right. \\
& \left.\left|F w_{0}\right|^{2} \leq 1+\left(\alpha^{2}-1\right) \beta^{2}, \quad\left|\operatorname{cof} F w_{0}\right|^{2} \leq \alpha^{2}-\left(\alpha^{2}-1\right) \beta^{2}\right\}
\end{aligned}
$$

Here and below $\lambda_{1}(F) \leq \lambda_{2}(F) \leq \lambda_{3}(F)$ denote the ordered singular values of $F$, i.e., the ordered eigenvalues of $\left(F^{T} F\right)^{1 / 2}$. 
Remark 2.2. In the case $\alpha \in(0,1)$ one obtains

$$
\begin{aligned}
K_{\mathrm{C}}^{\mathrm{qc}}= & \left\{F \in \mathbb{R}^{3 \times 3}: \operatorname{det} F=\alpha, \quad \lambda_{3}(F) \leq 1,\right. \\
& \left.\left|F w_{0}\right|^{2} \leq 1+\left(\alpha^{2}-1\right) \beta^{2}, \quad\left|\operatorname{cof} F w_{0}\right|^{2} \leq \alpha^{2}-\left(\alpha^{2}-1\right) \beta^{2}\right\} .
\end{aligned}
$$

In the following we focus on the physically-relevant case $\alpha>1$.

For a comparison, recall that the zero level set of the energy for (ideally soft) nematic elastomers reads, with the present notations,

$$
K_{\mathrm{N}}=S O(3)\left\{\mathrm{Id}+(\alpha-1) n \otimes n: n \in S^{2}\right\}=S O(3) \bigcup_{n \in S^{2}} U_{n} .
$$

Clearly, $K_{\mathrm{C}} \subset K_{\mathrm{N}}$; notice that $K_{\mathrm{N}}$ is five dimensional, while $K_{\mathrm{C}}$ is only four dimensional. This corresponds to the fact that $K_{\mathrm{N}}$ has full rotational symmetry, whereas $K_{\mathrm{C}}$ has only cylindrical symmetry. The quasiconvex hull of $K_{\mathrm{N}}$ is [11]

$$
K_{\mathrm{N}}^{\mathrm{qc}}=\left\{F \in \mathbb{R}^{3 \times 3}: \operatorname{det} F=\alpha, \quad \lambda_{1}(F) \geq 1\right\} .
$$

Clearly $K_{\mathrm{C}}^{\mathrm{qc}} \subset K_{\mathrm{N}}^{\mathrm{qc}}$, notice, however, that both sets are eight dimensional. In fact, even for the case of cubic-to-tetragonal phase transitions, where the corresponding set of energy-minimizing states $K_{\mathrm{ct}}$ is only three dimensional, the hull $K_{\mathrm{ct}}^{\mathrm{qc}}$ turns out to be eight dimensional [13]. The mechanical implication of this property is that, starting from a point in the relative interior of $K^{\mathrm{qc}}$, any small-enough volume-preserving deformation is soft.

We now turn to a more precise characterization of the microstructures leading to the relaxation result above. First we show that, for all matrices $F$ which are in the relative interior of $K_{\mathrm{C}}^{\mathrm{qc}}$, the variational problem

$$
\text { minimize } \int_{\Omega} W_{\mathrm{C}}(\nabla y) d x \text { : among all } y \text { such that } y(x)=F x \text { on } \partial \Omega
$$

has a Lipschitz-continuous minimizer, which obeys $W_{\mathrm{C}}(\nabla y)=0$ almost everywhere. In the proof we use the convex integration result by Müller and Šverák [18]; for alternative approaches to the same problem, see [10, 16].

Proposition 2.3. Under the same assumptions and in the notation of Theorem 2.1, let

$$
\begin{aligned}
F_{*} \in\left(K_{\mathrm{C}}^{\mathrm{qc}}\right)^{\mathrm{int}} & =\left\{F \in \mathbb{R}^{3 \times 3}: \operatorname{det} F=\alpha, \quad \lambda_{1}(F)>1,\right. \\
& \left.\left|F w_{0}\right|^{2}<1+\left(\alpha^{2}-1\right) \beta^{2}, \quad\left|\operatorname{cof} F w_{0}\right|^{2}<\alpha^{2}-\left(\alpha^{2}-1\right) \beta^{2}\right\} .
\end{aligned}
$$

Then, for any open domain $\Omega \subset \mathbb{R}^{3}$, the partial differential inclusion

$$
\begin{cases}\nabla y \in K_{\mathrm{C}} & \text { a.e. in } \Omega \\ y(x)=F_{*} x & \text { on } \partial \Omega\end{cases}
$$

has a Lipschitz solution $y: \Omega \rightarrow \mathbb{R}^{3}$. 


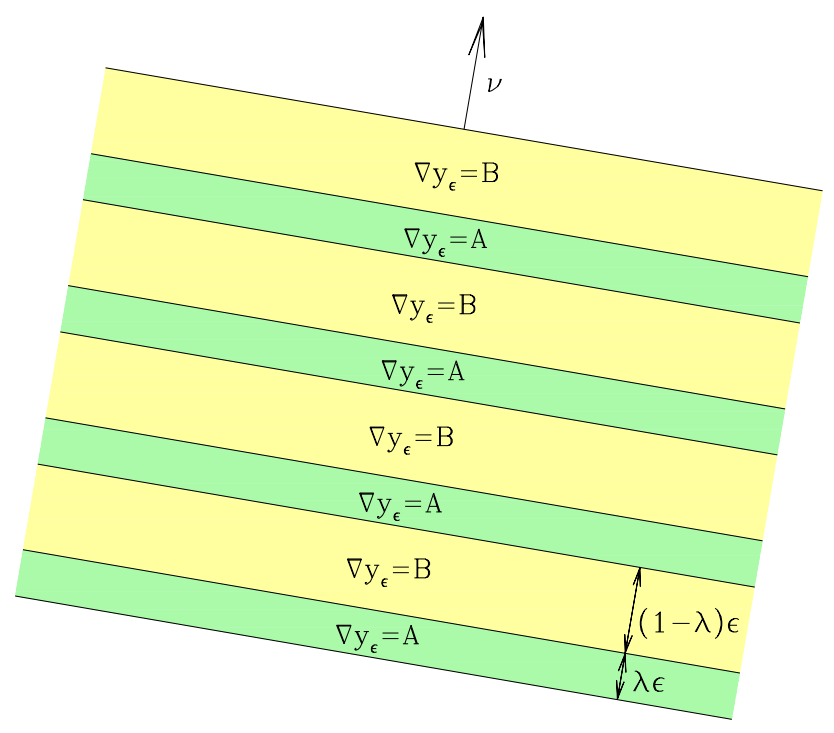

FIGURE 2: Schematic representation of the deformation in (2.6).

The Lipschitz maps $y$ constructed by convex integration have very complex geometric structures. Simpler patterns emerge by studying minimizing sequences in the form of laminates (see Figure 2). For example, a first-order laminate is obtained by considering deformations $y_{\varepsilon}$ whose gradients oscillate between two values, say $A$ and $B$, with volume fractions $\lambda$ and $1-\lambda$. The matrices $A$ and $B$ have to satisfy the condition $A-B=a \otimes \nu$, for some vectors $a \in \mathbb{R}^{3}$ and $\nu \in S^{2}$. The deformations have the form

$$
y_{\varepsilon}(x)=C x+a \varepsilon \chi\left(\frac{x \cdot \nu}{\varepsilon}\right),
$$

where $C=\lambda A+(1-\lambda) B$ is the average of the laminate, and $\chi$ is a 1-periodic function such that

$$
\chi(t)= \begin{cases}(1-\lambda) t & \text { if } 0 \leq t \leq \lambda \\ \lambda(1-t) & \text { if } \lambda \leq t \leq 1\end{cases}
$$

As $\varepsilon \rightarrow 0$, the deformation $y_{\varepsilon}$ approaches uniformly the affine map $C x$. Laminates of higher order are obtained by iterating this construction, see $[9,17,12]$. The laminates relevant for Theorem 2.1 are described in the following proposition. 
Proposition 2.4. Under the same assumptions and in the notation of Theorem 2.1,

(i) All matrices in $K_{\mathrm{C}}^{\mathrm{qc}}$ are averages of third-order laminates supported on $K_{\mathrm{C}}$.

(ii) All matrices in $K_{\mathrm{C}}^{\mathrm{qc}}$ where at least one of the three inequalities is an equality can be obtained as averages of second-order laminates supported on $K_{\mathrm{C}}$.

(iii) All matrices in $K_{\mathrm{C}}^{\mathrm{qc}}$ where two of the three inequalities are equalities, and only those, can be obtained as averages of first-order laminates supported on $K_{\mathrm{C}}$.

All proofs are given in Section 4.

\section{Physical and experimental implications}

Spontaneous deformations consisting of reversible shears of up to $\sim 20^{\circ}$ have been measured in smectic C elastomers [15], but no further mechanical experimental data is available. In comparison, smectic A elastomers have been studied in much greater detail: samples with the smectic layer compression modulus $(B$ in (2.4)) taking very large values $[20,19]$, or small values $[21,22]$ have been fabricated and mechanically tested. We propose here experimental geometries where soft elastic response and microstructure formation are expected for smectic $\mathrm{C}$ elastomers. We focus on a thin-film geometry since monodomain samples have only been obtained as thin films.

A mechanical experiment in which a specimen experiences a sequence of macroscopically affine deformations corresponds to a path through the space of matrices; in practice, one always starts from the cross-linking configuration, which lies on the boundary of $K_{\mathrm{C}}^{\mathrm{qc}}$. Thus, the path can either move away from $K_{\mathrm{C}}^{\mathrm{qc}}$, or can first move into $K_{\mathrm{C}}^{\mathrm{qc}}$ and eventually leave it later. In the ideal case, moving within $K_{\mathrm{C}}^{\mathrm{qc}}$ requires zero energy and therefore no stress. In practice, due to various sources of non-ideality in the rubber (e.g., a preferred direction from the two-stage crosslinking process), the stress cannot be exactly zero. One expects the signature of $K_{\mathrm{C}}^{\mathrm{qc}}$ to be a region which can be traversed with very small stress. For example, experiments on nematic elastomers have shown first a small, but rapid, increase in the stress, then a wide plateau with a much lower apparent elastic modulus, and finally a rapid increase in the stress after the plateau [25, Chapter 7.4].

In order to discuss experimental tests it is more convenient to work in a coordinate system in which the reference configuration is the one at cross 

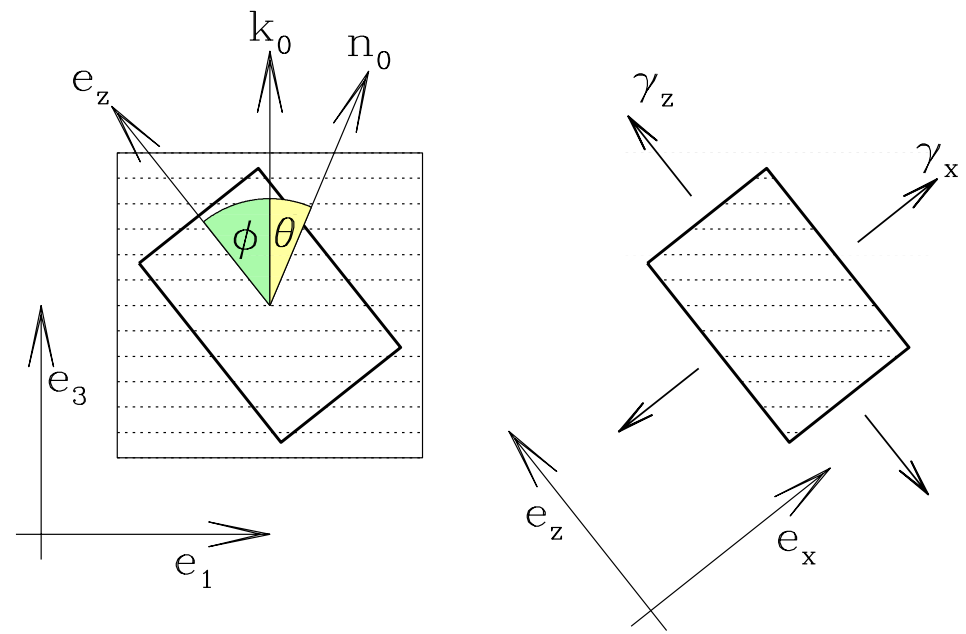

Figure 3: An illustration of the proposed experimental geometry, and of the coordinate system used. The relevant case has tension along $z$ (i.e., $\gamma_{z}>1$ ), and compression along $x$ (i.e., $\gamma_{x}<1$ ).

linking, which amounts to inverting the coordinate change discussed in the Appendix. Substituting $\Lambda=F U_{0}^{-1}$ into expression (2.4) shows that $F \in K_{\mathrm{C}}^{\mathrm{qc}}$ corresponds to $\Lambda \in K_{*}^{\mathrm{qc}}=K_{\mathrm{C}}^{\mathrm{qc}} U_{0}^{-1}$. Equivalently, $K_{*}^{\mathrm{qc}}$ is the set of matrices $\Lambda \in \mathbb{R}^{3 \times 3}$ satisfying the following relations:

$$
\begin{aligned}
\operatorname{det} \Lambda & =1 \\
\lambda_{1}\left(\Lambda U_{0}\right) & \geq 1 \\
\left|\Lambda U_{0}^{2} k_{0}\right|^{2} & \leq 1+\left(r^{2}-1\right) \cos ^{2} \theta, \\
\left|\Lambda^{-T} k_{0}\right|^{2} & \leq 1 .
\end{aligned}
$$

Here $r=\alpha^{2}, \theta$ is defined by

$$
\cos ^{2} \theta=\frac{\beta^{2}}{\alpha^{2}+\left(1-\alpha^{2}\right) \beta^{2}}
$$

and $k_{0}=U_{0}^{-1} w_{0} /\left|U_{0}^{-1} w_{0}\right|$.

The experimental configuration considered here is a thin film in the $\left(e_{1}, e_{3}\right)$ plane, layer normal $k_{0}=e_{3}$, and director tilted in the $e_{1}$ direction, i.e., $n_{0}=\sin \theta e_{1}+\cos \theta e_{3}$ (see Fig. 3).

\section{Shearing tests}

Consider deformations of the form

$$
\Lambda_{S}(\gamma)=\left(\begin{array}{ccc}
1 & 0 & \gamma \\
0 & 1 & 0 \\
0 & 0 & 1
\end{array}\right)
$$


which correspond to the geometry in which spontaneous shear was observed [15] when cooling from the smectic A to smectic C phase, because $\theta$ evolves from 0 to its current value. This matrix satisfies (3.4) and (3.2) with equality for $\gamma \leq 0$. The remaining inequality (3.3) then reduces to the following

$$
\left(1+(r-1) \cos ^{2} \theta\right)^{2}+(\gamma+(r-1) \cos \theta(\gamma \cos \theta+\sin \theta))^{2} \leq 1+\left(r^{2}-1\right) \cos ^{2} \theta
$$

We conclude that $\Lambda_{S}(\gamma) \in K_{*}^{\mathrm{qc}}$ provided that

$$
\gamma_{*} \leq \gamma \leq 0, \quad \text { where } \quad \gamma_{*}=-\frac{(r-1) \sin 2 \theta}{\sin ^{2} \theta+r \cos ^{2} \theta}
$$

In this case, the accompanying microstructure can also be determined explicitly. In particular, it is a simple laminate between $\Lambda_{S}(0)=\operatorname{Id}$ and $\Lambda_{S}\left(\gamma_{*}\right)$ with varying volume fractions, and with lamination planes parallel to the smectic layers, i.e., in the notation of (2.6), $B=\mathrm{Id}, A=\Lambda_{S}\left(\gamma_{*}\right), C=\Lambda(\gamma)$, and $\nu=k_{0}$, and $\lambda=\gamma / \gamma_{*}$. Therefore the nematic director initially has the value $n_{0}$ everywhere, and with increasing $\gamma$ it takes the value $n_{1}$ (corresponding to $\left.\Lambda_{S}\left(\gamma_{*}\right)\right)$ on larger and larger portions of the sample.

Notice that the matrices

$$
\left(\begin{array}{lll}
1 & 0 & 0 \\
0 & 1 & 0 \\
\gamma & 0 & 1
\end{array}\right),\left(\begin{array}{lll}
1 & 0 & 0 \\
0 & 1 & 0 \\
0 & \gamma & 1
\end{array}\right),\left(\begin{array}{lll}
1 & 0 & 0 \\
\gamma & 1 & 0 \\
0 & 0 & 1
\end{array}\right),\left(\begin{array}{lll}
1 & 0 & 0 \\
0 & 1 & \gamma \\
0 & 0 & 1
\end{array}\right),\left(\begin{array}{lll}
1 & \gamma & 0 \\
0 & 1 & 0 \\
0 & 0 & 1
\end{array}\right)
$$

do not give rise to soft deformations for $\gamma \neq 0$. The first two matrices do not satisfy (3.4), the third and fourth do not satisfy (3.3), and the fifth does not satisfy (3.2).

Simple shear testing of the sample may prove impractical to carry out experimentally as the sample may buckle for large imposed shears. Attributing any observed plateau in the stress-strain curve to soft deformation path may then be difficult. We now consider an experimental geometry in which the sample is under tension.

\section{Tensile tests}

Consider applying a biaxial extension to the film, with the direction of the largest stretch at an angle $\phi$ to the layer normal. This could be done, for example, by cutting out a small rectangular piece of elastomer from an existing film at the required angle to the layer normal and then applying biaxial stretches parallel to the sample edges ( $x$ and $z$ axes, see Fig. 3). We denote the rotated axes by $\left(e_{x}, e_{y}, e_{z}\right)$, to distinguish them from the original axes $\left(e_{1}, e_{2}, e_{3}\right)$. In practice we expect that the results here should also apply to 
uniaxial extension along the $e_{z}$ axis (and not only to the prescribed biaxial extension), as the other two directions should adjust so that the deformation remains in $K_{*}^{\mathrm{qc}}$. The deformation gradient for extension along $e_{z}$ is of the form

$$
\Lambda_{T}\left(\gamma_{x}, \gamma_{y}, \gamma_{z}\right)=\left(\begin{array}{ccc}
\gamma_{x} \cos ^{2} \phi+\gamma_{z} \sin ^{2} \phi & 0 & \left(\gamma_{x}-\gamma_{z}\right) \cos \phi \sin \phi \\
0 & \gamma_{y} & 0 \\
\left(\gamma_{x}-\gamma_{z}\right) \cos \phi \sin \phi & 0 & \gamma_{z} \cos ^{2} \phi+\gamma_{x} \sin ^{2} \phi
\end{array}\right)
$$

where $\gamma_{x}, \gamma_{y}$ and $\gamma_{z}$ are the principal stretches. We now calculate the range of values of $\phi$ for which paths of this form lie within $K_{*}^{\text {qc }}$. Due to the symmetry of $\Lambda_{T}$, it is only necessary to consider $0 \leq \phi \leq \pi / 2$.

Clearly by using (3.1) we obtain $\gamma_{y}=1 /\left(\gamma_{x} \gamma_{z}\right)$, hence we drop it from the notation. The remaining inequalities defining $K_{\mathrm{C}}^{\mathrm{qc}}$ show that $\Lambda_{T} \in K_{*}^{\mathrm{qc}}$ if the following conditions hold:

$$
\begin{aligned}
\frac{\sin ^{2} \phi}{\gamma_{x}^{2}}+\frac{\cos ^{2} \phi}{\gamma_{z}^{2}} \leq & 1 \\
0 \leq & 1+\gamma_{z}^{2} \gamma_{x}^{2} r-\gamma_{x}^{2}\left(\cos ^{2}(\phi+\theta)+r \sin ^{2}(\phi+\theta)\right) \\
& -\gamma_{z}^{2}\left(\sin ^{2}(\phi+\theta)+r \cos ^{2}(\phi+\theta)\right), \\
\gamma_{x} \gamma_{z} \leq & 1, \\
1+\left(r^{2}-1\right) \cos ^{2} \theta \geq & \gamma_{z}^{2}\left(\frac{r+1}{2} \cos \phi+\frac{r-1}{2} \cos (\phi+2 \theta)\right)^{2} \\
& +\gamma_{x}^{2}\left(\frac{r+1}{2} \sin \phi+\frac{r-1}{2} \sin (\phi+2 \theta)\right)^{2} .
\end{aligned}
$$

Here (3.5) arises from the condition (3.4), (3.6) and (3.7) arise from (3.2), and (3.8) arises from (3.3). An illustration of the set of pairs $\left(\gamma_{x}, \gamma_{z}\right)$ satisfying these four inequalities is given in Fig. 4. All four conditions are equalities for $\Lambda_{T}(1,1)=\mathrm{Id}$, corresponding to the fact that this matrix is on the boundary of $K_{*}^{\mathrm{qc}}$.

We now check if, for suitable choices of the angle $\phi$, there are curves in the $\left(\gamma_{x}, \gamma_{z}\right)$ plane which stay within $K_{*}^{\mathrm{qc}}$. To do this, we compute the slopes of the four curves given above, and compare their values at the point $(1,1)$. It turns out that there is a nonempty set which satisfies all four conditions only if

$$
\frac{\pi}{4}-\theta+\frac{1}{2} \arctan \frac{\tan \theta}{r} \leq \phi \leq \frac{\pi}{4}
$$

Therefore soft elasticity is expected only for samples with orientation in this particular range. 


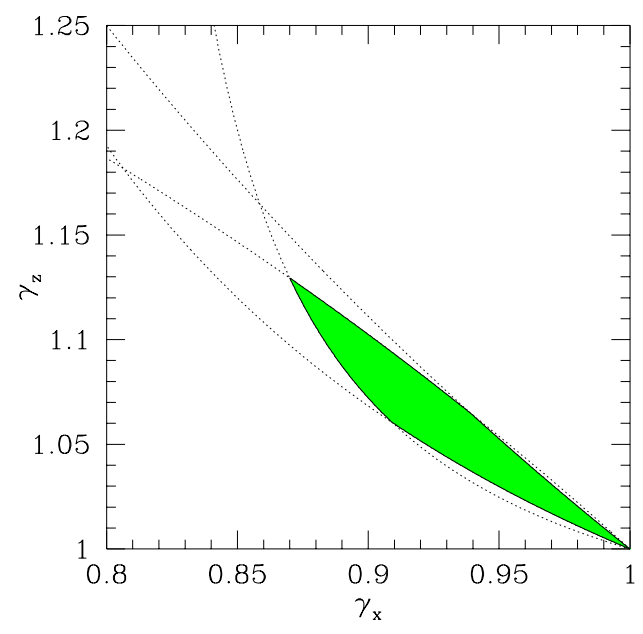

(a)

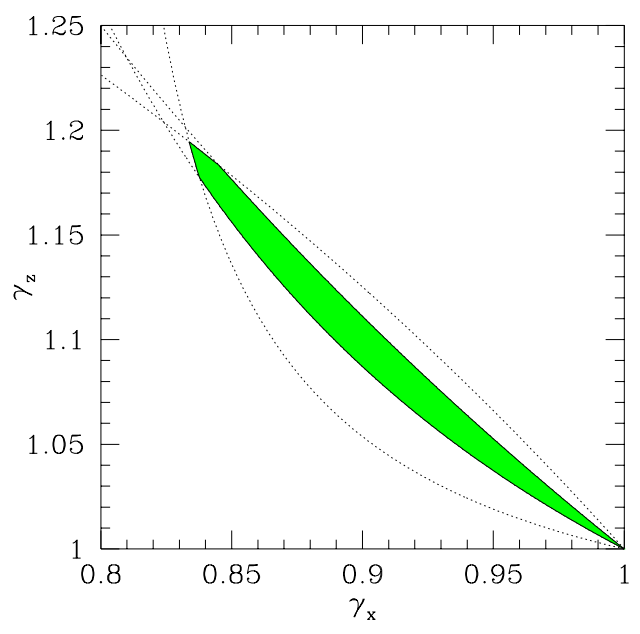

(b)

FIGURE 4: Illustration of the stretches $\gamma_{x}, \gamma_{z}$ for which the deformation $\Lambda_{T}$ is soft (i.e., in $K_{*}^{\mathrm{qc}}$ ), for $r=2$ and $\theta=22.5^{\circ}$. (a) stretching direction $\phi=36^{\circ}$, (b) stretching direction $\phi=39^{\circ}$.

We are now interested in the amount of soft deformation that can be sustained by the sample. Precisely, for any value of $\phi$ we compute the maximal soft stretch in the $z$ direction,

$$
\gamma_{z}^{\max }=\max \left\{\gamma_{z}: \quad \Lambda_{T}\left(\gamma_{x}, \gamma_{z}\right) \in K_{*}^{\mathrm{qc}} \text { for some } \gamma_{x}\right\}
$$

Computing $\gamma_{z}^{\max }$ involves solving several algebraic equations, corresponding to the various pairs of inequalities in (3.5)-(3.8). The results are reported in Figure 5 for some realistic values of the parameters.

\section{Proofs of the results in Section 2}

We start by recalling a result on the relaxation of the two-well problem [4, 24]. Its proof is given at the end of this section for the convenience of the reader.

Lemma 4.1. For any pair $n_{1}, n_{2} \in S^{2}$, the hull of the two wells

$$
K_{12}=S O(3) U_{n_{1}} \cup S O(3) U_{n_{2}}
$$

is given by

$$
\begin{aligned}
K_{12}^{\mathrm{qc}}= & \left\{F \in \mathbb{R}^{3 \times 3}: \operatorname{det} F=\alpha, \alpha F v=\operatorname{cof} F v\right. \\
& \left.\left|F f_{1}\right| \leq\left|U_{n_{1}} f_{1}\right|,\left|F f_{2}\right| \leq\left|U_{n_{1}} f_{2}\right|\right\}
\end{aligned}
$$




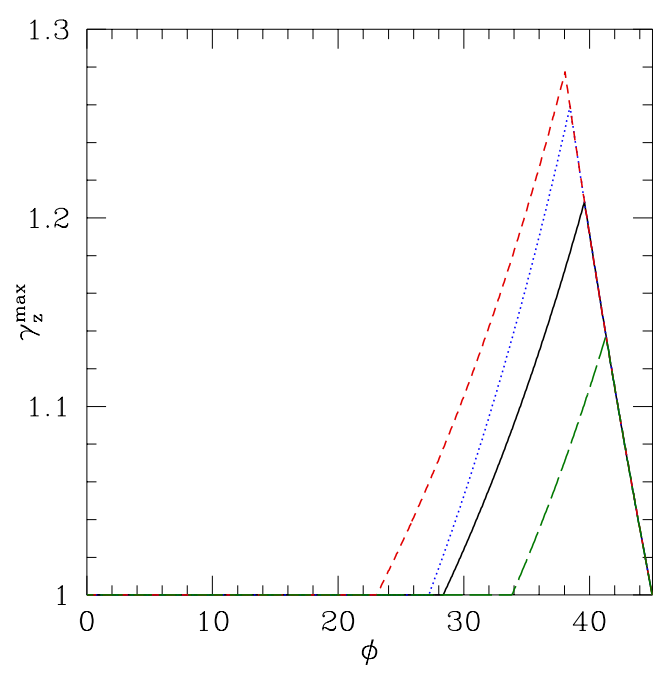

FigURE 5: An illustration of the expected amplitude of the soft plateau $\gamma_{z}^{\max }$ as a function of orientation angle $\phi$ (in degrees). Full (black) curve, $r=2$ and $\theta=22.5^{\circ}$; dotted (blue) curve, $r=2.5$ and $\theta=22.5^{\circ}$; short-dashed (red) curve, $r=2$ and $\theta=30^{\circ}$; long-dashed (green) curve, $r=2$ and $\theta=15^{\circ}$.

Here

$$
v=\frac{n_{1} \wedge n_{2}}{\left|n_{1} \wedge n_{2}\right|}, \quad f_{1}=\frac{n_{1}+n_{2}}{\left|n_{1}+n_{2}\right|}, \quad f_{2}=\frac{n_{1}-n_{2}}{\left|n_{1}-n_{2}\right|} .
$$

The set $K_{12}^{\mathrm{qc}}$ is polyconvex; each matrix in $K_{12}^{\mathrm{qc}}$ is the average of a secondorder laminate supported on $K_{12}$; the set of matrices which are averages of first-order laminates supported on $K_{12}$ is the subset of $K_{12}^{\mathrm{qc}}$ where one of the two inequalities is an equality.

Geometrically, $v$ is the normal to the plane spanned by $n_{1}$ and $n_{2}$; the vectors $\left(v, f_{1}, f_{2}\right)$ form an orthonormal basis, and the $f_{i}$ are (up to a sign) the only two unit vectors orthogonal to $v$ which fulfill $\left|U_{n_{1}} f\right|=\left|U_{n_{2}} f\right|$. Further, since $\operatorname{det} F=\alpha$ the condition $\alpha F v=\operatorname{cof} F v$ is equivalent to $F^{T} F v=v$. This implies, in particular, that one of the singular values of $F$ is 1 .

Proof of Theorem 2.1. Let $\widetilde{K}$ be the set in the right-hand side of (2.4), i.e., the set of matrices $F \in \mathbb{R}^{3 \times 3}$ such that

$$
\begin{aligned}
\operatorname{det} F & =\alpha \\
\left|F w_{0}\right|^{2} & \leq 1+\left(\alpha^{2}-1\right) \beta^{2}, \\
\left|\operatorname{cof} F w_{0}\right|^{2} & \leq \alpha^{2}-\left(\alpha^{2}-1\right) \beta^{2}, \\
\lambda_{1}(F) & \geq 1 .
\end{aligned}
$$


The set $\widetilde{K}$ is clearly polyconvex, as it is defined as the intersection of sublevel sets of polyconvex functions (recall that $\lambda_{1}(F)=\operatorname{det} F / \lambda_{3}(\operatorname{cof} F)$, hence (4.6) can be equivalently replaced by $\left.\lambda_{3}(\operatorname{cof} F) \leq \alpha\right)$. Therefore it suffices to show that any matrix in $\widetilde{K}$ is the average of a laminate supported on $K$. Due to the rotational invariance, it further suffices to consider symmetric matrices in $\widetilde{K}$.

We decompose $\widetilde{K}$ into three sets $\widetilde{K}^{(i)}, i=1,2,3$. In the first one all three inequalities are equalities,

$$
\begin{gathered}
\widetilde{K}^{(1)}:=\left\{F \in \widetilde{K}: \lambda_{1}(F)=1, \quad\left|F w_{0}\right|^{2}=1+\left(\alpha^{2}-1\right) \beta^{2},\right. \\
\left.\left|\operatorname{cof} F w_{0}\right|^{2}=\alpha^{2}-\left(\alpha^{2}-1\right) \beta^{2}\right\} .
\end{gathered}
$$

The second one contains matrices with one singular value equal to 1 ,

$$
\widetilde{K}^{(2)}:=\left\{F \in \widetilde{K}: \lambda_{1}(F)=1\right\}
$$

The third one contains matrices with all singular values strictly larger than 1 ,

$$
\widetilde{K}^{(3)}:=\left\{F \in \widetilde{K}: \lambda_{1}(F)>1\right\}
$$

We claim that

(i) $\widetilde{K}^{(1)}=K_{\mathrm{C}}$;

(ii) all matrices in $\widetilde{K}^{(2)}$ are averages of second-order laminates supported on two wells in $K_{\mathrm{C}}$;

(iii) all matrices in $\widetilde{K}^{(3)}$ are averages of simple laminates supported in $\widetilde{K}^{(2)}$.

Since $\widetilde{K}=\widetilde{K}^{(2)} \cup \widetilde{K}^{(3)}$, this will imply the thesis. It remains to prove the three claims. For notational simplicity, in the following we work with $w_{0}=e_{3}$.

Proof of Claim (iii). Let $F \in \widetilde{K}^{(3)}$, and consider the rank-one line

$$
F_{t}=F\left(\mathrm{Id}+t e_{1} \otimes e_{2}\right) .
$$

To prove the claim it suffices to determine two values of $t$, say $t_{+}>0>t_{-}$, such that $F$ can be written as an average of $F_{t_{+}}$and $F_{t_{-}}$, with $F_{t_{ \pm}} \in \widetilde{K}^{(2)}$. To do this, we first compute

$$
\operatorname{cof} F_{t}=\operatorname{cof} F\left(\operatorname{Id}-t e_{2} \otimes e_{1}\right), \quad \text { and } \quad \operatorname{det} F_{t}=\alpha .
$$

Therefore the quantities $\operatorname{det} F_{t},\left|F_{t} e_{3}\right|$, and $\left|\operatorname{cof} F_{t} e_{3}\right|$ do not depend on $t$. Consider now the function

$$
f(t)=\lambda_{1}\left(F_{t}\right)
$$


This is continuous, $f(0)>1$, and $\lim _{t \rightarrow \pm \infty} f(t)=0$. To see the latter, it suffices to observe that

$$
\infty=\lim _{t \rightarrow \pm \infty}\left|F_{t}\right|^{2}=\lim _{t \rightarrow \pm \infty} \lambda_{3}^{2}\left(F_{t}\right)+\lambda_{2}^{2}\left(F_{t}\right)+\lambda_{1}^{2}\left(F_{t}\right) \leq \lim _{t \rightarrow \pm \infty} \frac{3 \alpha^{2}}{\lambda_{1}^{4}\left(F_{t}\right)}
$$

since $\operatorname{det} F_{t}=\alpha$ implies $\lambda_{3}\left(F_{t}\right)=\alpha / \lambda_{2}\left(F_{t}\right) \lambda_{1}\left(F_{t}\right) \leq \alpha / \lambda_{1}^{2}\left(F_{t}\right)$. We conclude that the equation $f(t)=1$ has at least one positive and one negative solution (actually they are unique, since $\lambda_{1}$ is concave along volume-preserving rankone directions). The solutions are the desired $t_{+}$and $t_{-}$.

Proof of Claim (ii) We can assume without loss of generality that $F$ is symmetric, so that $\lambda_{1}(F)=1$ means that $F$ has an eigenvalue 1 . Let $v$ be the corresponding eigenvector. Exploiting the cylindrical symmetry we can assume that $v \cdot e_{2}=0$, and obtain

$$
F v=v \quad \text { for } \quad v=\left(\begin{array}{c}
\cos \phi \\
0 \\
\sin \phi
\end{array}\right) .
$$

We intend to determine values of $n_{1}$ and $n_{2} \in N_{\mathrm{C}}$ such that we can use Lemma 4.1, with this vector $v$. It is convenient to start from the two vectors $f_{i}$, as in (4.2). Indeed, $f_{1}$ is a unit vector normal to both $w_{0}$ and $v$, hence $f_{1}=e_{2}$; in turn, $f_{2}$ is normal to both $f_{1}$ and $v$. In components, we obtain

$$
f_{1}=\left(\begin{array}{l}
0 \\
1 \\
0
\end{array}\right), \quad \text { and } \quad f_{2}=\left(\begin{array}{c}
-\sin \phi \\
0 \\
\cos \phi
\end{array}\right) \text {. }
$$

We now claim that $F \in \widetilde{K}$ implies

$$
\left|F f_{1}\right|^{2} \leq 1+\left(\alpha^{2}-1\right)\left(1-\frac{\beta^{2}}{\cos ^{2} \phi}\right)
$$

and

$$
\left|F f_{2}\right|^{2} \leq 1+\left(\alpha^{2}-1\right) \frac{\beta^{2}}{\cos ^{2} \phi} .
$$

Since $1 \leq \lambda_{1}(F) \leq\left|F f_{1}\right|$, (4.8) implies that $\beta^{2} \leq \cos ^{2} \phi$. We can therefore define

$$
n_{1,2}=\left(\begin{array}{c}
-\beta \tan \phi \\
\pm \sqrt{1-\beta^{2} / \cos ^{2} \phi} \\
\beta
\end{array}\right) \in K_{\mathrm{C}} .
$$

These vectors have been determined so that (4.2) is satisfied. In turn, the expressions on the right-hand side of (4.8) and (4.9) are exactly the values 
of $\left|U_{n_{1}} F_{i}\right|^{2}=\left|U_{n_{2}} F_{i}\right|^{2}$. Therefore by Lemma 4.1 (4.8) and (4.9) imply that $F$ is in the second lamination convex hull of $S O(3)\left\{U_{n_{1}}, U_{n_{2}}\right\} \subset K_{\mathrm{C}}$.

It remains to prove the claimed (4.8)-(4.9). To do so, we write

$$
w_{0}=\sin \phi v+\cos \phi f_{2},
$$

and observe that $\{v\},\{v\}^{\perp}$ are eigenspaces of $F$. Therefore,

$$
\left|F w_{0}\right|^{2}=\sin ^{2} \phi|F v|^{2}+\cos ^{2} \phi\left|F f_{2}\right|^{2},
$$

and using (4.7) and (4.4) we get (4.9). Analogously,

$$
\left|\operatorname{cof} F w_{0}\right|^{2}=\sin ^{2} \phi|\operatorname{cof} F v|^{2}+\cos ^{2} \phi\left|\operatorname{cof} F f_{2}\right|^{2} .
$$

Using (4.7) and (4.5) we get

$$
\left|\operatorname{cof} F f_{2}\right|^{2} \leq \alpha^{2}-\left(\alpha^{2}-1\right) \frac{\beta^{2}}{\cos ^{2} \phi} .
$$

We recall that if $F^{T} F v=v$, then

$$
|F \mu|=|\operatorname{cof} F(v \wedge \mu)| \quad \text { for all } \mu \in \mathbb{R}^{3}, \quad \mu \cdot v=0 .
$$

To see this, it suffices to verify it for symmetric $F$, so that $v$ is an eigenvector with eigenvalue 1 . Then, $\operatorname{cof} F(v \wedge \mu)=F v \wedge F \mu=v \wedge F \mu$, and the two vectors are orthogonal. Using (4.11) in (4.10), we obtain (4.8).

Proof of Claim (i) If $F \in K_{\mathrm{C}}$, then $F=Q U_{n}$ for some $n \in N_{\mathrm{C}}$, hence

$$
\left|F w_{0}\right|^{2}=\left|U_{n} w_{0}\right|^{2}=1+\left(\alpha^{2}-1\right)\left(n \cdot w_{0}\right)^{2}=1+\left(\alpha^{2}-1\right) \beta^{2}
$$

and

$$
\left|\operatorname{cof} F w_{0}\right|^{2}=\left|\alpha U_{n}^{-1} w_{0}\right|^{2}=\alpha^{2}+\left(1-\alpha^{2}\right)\left(n \cdot w_{0}\right)^{2}=\alpha^{2}+\left(1-\alpha^{2}\right) \beta^{2},
$$

therefore $F \in \widetilde{K}^{(1)}$.

If $F \in \widetilde{K}^{(1)} \subset \dot{\widetilde{K}}^{(2)}$, then we can repeat the argument given in the proof of Claim (ii), and obtain that equality holds in (4.8) and (4.9). Since $\left(v, f_{1}, f_{2}\right)$ is an orthonormal basis,

$$
|F|^{2}=|F v|^{2}+\left|F f_{1}\right|^{2}+\left|F f_{2}\right|^{2}=2+\alpha^{2} .
$$

Recalling that $\operatorname{det} F=\alpha$ and $\lambda_{1}(F)=1$, we have

$$
\lambda_{2}(F) \lambda_{3}(F)=\alpha, \quad \lambda_{2}^{2}(F)+\lambda_{3}^{2}(F)=1+\alpha^{2},
$$

hence $\lambda_{2}(F)=1, \lambda_{3}(F)=\alpha$. Therefore $F=Q U_{n}$, for some $n \in S^{2}$. Finally,

$$
\left|F w_{0}\right|^{2}=\left|U_{n} w_{0}\right|^{2}=1+\left(\alpha^{2}-1\right)\left(n \cdot w_{0}\right)^{2},
$$

therefore $\left(n \cdot w_{0}\right)^{2}=\beta^{2}$. Replacing if necessary $n$ with $-n$, we conclude that $n \cdot w_{0}=\beta$, hence $n \in N_{\mathrm{C}}$ and $F \in K_{\mathrm{C}}$. 
Proof of Remark 2.2. The proof follows the same steps as the one of Theorem 2.1, replacing the condition $\lambda_{1} \geq 1$ with the condition $\lambda_{3} \leq 1$. In particular, to show that $\beta^{2} \leq \cos ^{2} \phi$ (after (4.8)), one uses (4.9) and the fact that $\lambda_{3} \leq 1$.

Proof of Proposition 2.4. Part (i) follows from the claims (ii) and (iii) in the proof of Theorem 2.1.

We now prove part (iii). First-order laminates only involve two values of the director $n$, hence by Lemma 4.1 their average satisfies $\lambda_{1}(F)=1$, and one of the two inequalities in (4.1) is an equality. Therefore also one of the other two inequalities in (2.4) is an equality, see proof of Claim (i) above.

Conversely, assume that $\lambda_{1}(F)=1$, and that one of the other inequalities in (2.4) is an equality. Then, argueing as in the proof of Theorem 2.1, we obtain that either (4.8) or (4.9) is an equality. Therefore, application of Lemma 4.1 as in the proof of Claim (ii) above gives a first-order laminate with average $F$.

Finally, assume that

$$
\left|F w_{0}\right|^{2}=1+\left(\alpha^{2}-1\right) \beta^{2} \quad \text { and } \quad\left|\operatorname{cof} F w_{0}\right|^{2}=\alpha^{2}-\left(\alpha^{2}-1\right) \beta^{2},
$$

and consider the construction used in the proof of Claim (iii). The matrices $F_{t}$ constructed there belong to $\widetilde{K}^{(1)}=K_{\mathrm{C}}$.

It remains to show part (ii) of Proposition 2.4. If $\lambda_{1}(F)=1$, then $F \in$ $\widetilde{K}^{(2)}$, hence it is the average of a second-order laminate by Claim (ii). If instead one of the other inequalities is an equality, we consider a first-order laminate as in the proof of Claim (iii) above. For the resulting matrices two inequalities are equalities, hence by Part (iii) (proven above) are the average of first-order laminates. This concludes the proof.

Proof of Proposition 2.3. Proposition 2.3 is proven on the basis of the convex integration result by Müller and Šverák [18, Theorem 1.3], by constructing a suitable in-approximation for the set $K_{\mathrm{C}}$ (see (4.14) below for a definition). This will be done by considering a family of variants of the set $K_{\mathrm{C}}$ which interpolates between $S O(3)$ and the original set $K_{\mathrm{C}}$. To do this, it is necessary to work with constant determinant, and it is convenient to make the dependence on $\alpha$ and $\beta$ explicit in the notation. We change variables according to

$$
\widetilde{F}=\alpha^{-1 / 3} F,
$$

and write

$$
\widetilde{K}_{\alpha, \beta}=S O(3)\left\{\alpha^{-1 / 3} \mathrm{Id}+\left(\alpha^{2 / 3}-\alpha^{-1 / 3}\right) n \otimes n: n \in N_{\mathrm{C}}^{\beta}\right\},
$$


where $N_{\mathrm{C}}^{\beta}$ is defined as in (2.3). The hull can be characterized as

$$
\widetilde{K}_{\alpha, \beta}^{\mathrm{qc}}=\left\{F \in \mathbb{R}^{3 \times 3}: \operatorname{det} F=1, \psi_{j}(F) \leq \phi_{j}(\alpha, \beta), \quad j=1,2,3\right\}
$$

where

$$
\begin{aligned}
\psi_{1}(F)=\frac{1}{\lambda_{1}(F)}, & \phi_{1}(\alpha, \beta)=\alpha^{1 / 3}, \\
\psi_{2}(F)=\left|F w_{0}\right|^{2}, & \phi_{2}(\alpha, \beta)=\alpha^{-2 / 3}+\left(\alpha^{4 / 3}-\alpha^{-2 / 3}\right) \beta^{2}, \\
\psi_{3}(F)=\left|\operatorname{cof} F w_{0}\right|^{2}, & \phi_{3}(\alpha, \beta)=\alpha^{2 / 3}-\left(\alpha^{2 / 3}-\alpha^{-4 / 3}\right) \beta^{2} .
\end{aligned}
$$

By Claim (i) in the proof of Theorem 2.1, replacing inequalities with equalities this reduces to $\widetilde{K}_{\alpha, \beta}$, i.e.,

$$
\widetilde{K}_{\alpha, \beta}=\left\{F \in \mathbb{R}^{3 \times 3}: \operatorname{det} F=1, \psi_{j}(F)=\phi_{j}(\alpha, \beta) j=1,2,3\right\} .
$$

We want to construct an in-approximation, i.e., a sequence of uniformly bounded, relatively open sets $U_{i} \subset\left\{F \in \mathbb{R}^{3 \times 3}: \operatorname{det} F=1\right\}$ such that

$$
U_{i} \subset\left(U_{i+1}\right)^{\mathrm{rc}}, \quad \text { and } \quad U_{i} \rightarrow \widetilde{K} .
$$

Here $U_{i} \rightarrow \widetilde{K}$ means that $F_{i} \rightarrow F, F_{i} \in U_{i}$ implies $F \in \widetilde{K} ; U^{\text {rc }}$ denotes the rank-one convex hull of $U$, see $[9,17,12]$. We claim that we can choose $\alpha_{i}>1, \beta_{i} \in(0,1), \varepsilon_{i}>0$ such that

$$
U_{i}=\left\{F: \operatorname{det} F=1, \operatorname{dist}\left(F, \widetilde{K}_{\alpha_{i}, \beta_{i}}\right)<\varepsilon_{i}\right\}
$$

satisfies (4.14). These sets are relatively open, uniformly bounded, and the convergence to $\widetilde{K}$ is ensured provided that

$$
\alpha_{i} \rightarrow \alpha, \quad \beta_{i} \rightarrow \beta, \quad \text { and } \quad \varepsilon_{i} \rightarrow 0 \text {. }
$$

We shall choose $\alpha_{i}$ and $\beta_{i}$ so that

$$
\widetilde{K}_{\alpha_{i}, \beta_{i}} \subset\left(\widetilde{K}_{\alpha_{i+1}, \beta_{i+1}}^{\mathrm{rc}}\right)^{\mathrm{int}}
$$

Here $A^{\text {int }}$ denotes the relative interior of the set $A \subset\{F: \operatorname{det} F=1\}$. Since the first set in (4.15) is compact and the second relatively open, if (4.15) holds then we can choose $\varepsilon_{i}>0$ such that (4.14) holds.

It remains to choose $\alpha_{i}$ and $\beta_{i}$ so that (4.15) holds. Since by Proposition $2.4 \widetilde{K}_{\alpha_{i+1}, \beta_{i+1}}^{\mathrm{rc}}=\widetilde{K}_{\alpha_{i+1}, \beta_{i+1}}^{\mathrm{qc}}$, recalling (4.12) and (4.13), (4.15) is equivalent to the condition

$$
\phi_{j}\left(\alpha_{i}, \beta_{i}\right)<\phi_{j}\left(\alpha_{i+1}, \beta_{i+1}\right) \quad j=1,2,3 .
$$


We claim that there is a curve $\gamma$ starting from the point $(\alpha, \beta)$ and such that the three functions $\phi_{j} \circ \gamma$ are strictly decreasing along $\gamma$. If this is the case, it suffices to choose appropriately the points along $\gamma$ to obtain sets fulfilling (4.15). Precisely, assume

$$
\gamma(0)=(\alpha, \beta), \quad \nabla \phi_{j} \circ \gamma \cdot \gamma^{\prime}(t)<0 \text { for } t \in\left[0, t_{0}\right], t_{0}>0 .
$$

(here $(\alpha, \beta)$ denote the values entering the statement, i.e., the parameters in $(2.5))$. Choose $t_{1} \in\left(0, t_{0}\right)$ such that $\psi_{j}\left(F_{*}\right)<\phi_{j}\left(\gamma\left(t_{0}\right)\right)$ for all $j$, which is possible since by assumption $\phi_{j}\left(F_{*}\right)<\phi_{j}(\gamma(0))$ for all $j$. Then, the points

$$
\left(\alpha_{i}, \beta_{i}\right)=\gamma\left(2^{-i} t_{1}\right)
$$

satisfy all requirements.

It remains to show existence of the curve $\gamma$ with the properties given in (4.16). We prove this by showing existence of a vector field along which all $\nabla \phi_{j}$ are negative. For each $(\alpha, \beta)$, the set of directions which have a negative component along $\nabla \phi_{2}$ is an open half-plane. The same holds for $\nabla \phi_{3}$. Since the two gradients do not sum to zero, the two half-planes intersect in a cone, and there is a direction on which both $\phi_{2}$ and $\phi_{3}$ are decreasing. We claim that in this direction also $\phi_{1}$ is decreasing. Indeed,

$$
\nabla \phi_{2}+\alpha^{2 / 3} \nabla \phi_{3}=\left(\begin{array}{c}
\frac{2}{3}\left(1+\beta^{2}\right) \alpha^{-5 / 3}\left(\alpha^{2}-1\right) \\
0
\end{array}\right) .
$$

Therefore

$$
\nabla \phi_{1}=\frac{\alpha}{2\left(\alpha^{2}-1\right)\left(\beta^{2}+1\right)}\left[\nabla \phi_{2}+\alpha^{2 / 3} \nabla \phi_{3}\right]
$$

Since the fraction is positive, we conclude that if $\phi_{2}$ and $\phi_{3}$ are decreasing, so is $\phi_{1}$.

Proof of Lemma 4.1. Let $\widetilde{K}$ denote the right-hand side of (4.1). This set is clearly polyconvex. Further, $K_{12} \subset \widetilde{K}$. Even more, we claim that $K_{12}$ equals the set of matrices in $\widetilde{K}$ where both inequalities are equalities. To see this, assume

$$
\operatorname{det} F=\alpha, \quad \alpha F v=\operatorname{cof} F v, \quad\left|F f_{1}\right|=\left|U_{n_{1}} f_{1}\right|, \quad\left|F f_{2}\right|=\left|U_{n_{1}} f_{2}\right| .
$$

This implies

$$
|F|^{2}=\left|U_{n_{1}}\right|^{2}=2+\alpha^{2} .
$$

Therefore the singular values of $F$ are $(1,1, \alpha)$, i.e., $F=Q U_{n}$, for some $n \in S^{2}$. Clearly $v \cdot n=0$. Further,

$$
\left(n \cdot f_{i}\right)^{2}=\left(n_{1} \cdot f_{i}\right)^{2}=\left(n_{2} \cdot f_{i}\right)^{2} \quad i=1,2 .
$$


Therefore $n \in\left\{ \pm n_{1}, \pm n_{2}\right\}$ and $F \in K_{12}$.

Let now $F \in \widetilde{K}$ be such that

$$
\left|F f_{1}\right|=\left|U_{n_{1}} f_{1}\right|, \quad\left|F f_{2}\right|<\left|U_{n_{1}} f_{2}\right| .
$$

the other case being equivalent. We consider the rank-one line

$$
F_{t}=F\left(\mathrm{Id}+t f_{1} \otimes f_{2}\right) .
$$

We notice that $\operatorname{det} F_{t}, F_{t} v$, cof $F_{t} v$ and $F_{t} f_{1}$ do not depend on $t$. At the same time, $f(t)=\left|F_{t} f_{2}\right|$ is continuous, and diverges as $t \rightarrow \pm \infty$, hence there are two values $t_{-}<0<t_{+}$such that $f\left(t_{ \pm}\right)=\left|U_{n_{1}} f_{2}\right|$. For the matrices $F_{t_{ \pm}}$all inequalities are equalities, hence by the previous argument they are in $K_{12}$, and $F$ is the average of a first-order laminate supported on them.

Finally, for a generic $F \in \widetilde{K}$ we consider the rank-one line

$$
F_{t}=F\left(\operatorname{Id}+t f_{2} \otimes f_{1}\right) .
$$

Reasoning as above, we can find two values of $t$ such that

$$
\left|F_{t_{ \pm}} f_{1}\right|=\left|U_{n_{1}} f_{1}\right|
$$

and reduce to the previous case. The conclusion follows.

\section{Discussion and outlook}

In summary, we have determined the quasiconvex hull of the zero set of the energy density for smectic $\mathrm{C}$ elastomers derived in [2], and have shown that it contains a full-dimensional set of volume-preserving zero-energy deformations. We used our result to predict microstructure formation and soft elastic response in stretching experiments on thin sheets (see Fig. 5), and to propose experimental geometries that should make the effect visible.

In the related case of smectic A elastomers, which corresponds to taking $\beta=1$, one obtains as zero set of the energy $K_{\mathrm{A}}=S O(3) U_{w_{0}}$ (see [1]). Therefore the quasiconvex hull is trivial, $K_{\mathrm{A}}^{\mathrm{qc}}=K_{\mathrm{A}}$. The mechanical interpretation of this fact is that there are no zero-energy soft modes. Microstructure formation and softening at intermediate stretches have, however, been observed experimentally [19] for stretches along the layer normal, and explained theoretically [1] as due to buckling of the smectic layers. Similar and more complex effects can be expected in smectic C.

The analysis in [1] can be recast in the present variational framework, leading to a partial determination of the quasiconvex envelope of the smectic 
A energy density. This shows that there exists a large variety of physicallyrelevant regimes outside of the zero-energy set, and that the knowledge of the full quasiconvex envelope of the energy density may shed further light on the complex physics of these interesting materials. The determination of the envelope for the smectic A energy density is, in the general case, open, and even more so for smectic C.

\section{Acknowledgements}

This work was partially supported by Deutsche Forschungsgemeinschaft through the Schwerpunktprogramm 1095 Analysis, Modeling and Simulation of Multiscale Problems and by the Istituto Nazionale di Alta Matematica through the research project Mathematical Challenges in Nanomechanics.

\section{A Appendix}

In [2], the following energy density is derived from a statistical mechanical model

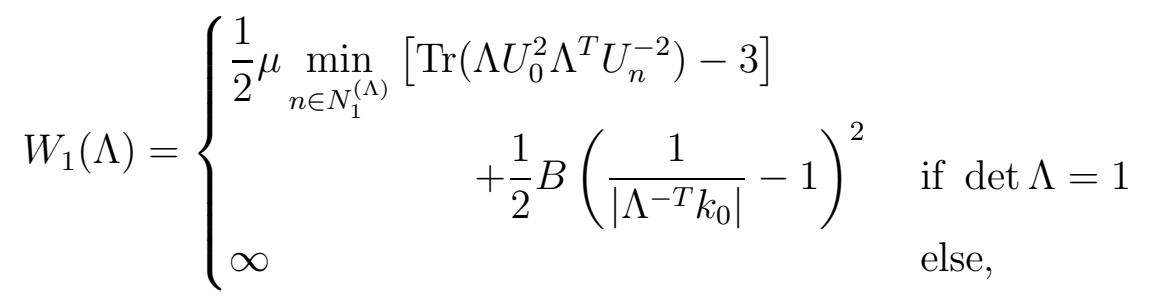

where $\Lambda \in \mathbb{R}^{3 \times 3}$ is the deformation gradient with respect to the cross-linking configuration, $k_{0}$ is the unit normal to the smectic layers at cross-linking, and the smectic director $n$ is a unit vector is the set

$$
N_{1}^{(\Lambda)}=\left\{n \in S^{2}: n \cdot \frac{\Lambda^{-T} k_{0}}{\left|\Lambda^{-T} k_{0}\right|}=\cos \theta\right\} .
$$

Further,

$$
U_{n}=\mathrm{Id}+(\alpha-1) n \otimes n
$$

is a uniaxial stretch along $n$, and $U_{0}=U_{n_{0}}, n_{0} \in N_{1}^{(\mathrm{Id})}$ being the director at cross-linking. The expressions in [2] are written in terms of $r=\alpha^{2}$ and $\ell_{n}=U_{n}^{2}$.

As in the case of nematic elastomers, it is convenient to change reference configuration, in order to fully exploit the symmetry of the problem. In particular, we replace the variable $\Lambda$ with $F$, defined by

$$
F=\Lambda U_{0}=\Lambda\left(\mathrm{Id}+(\alpha-1) n_{0} \otimes n_{0}\right),
$$


and correspondingly

$$
\begin{aligned}
W_{\mathrm{C}}(F)= & W_{1}\left(F U_{0}^{-1}\right) \\
= & \begin{cases}\frac{1}{2} \mu \min _{n \in N_{2}^{(F)}}\left[|F|^{2}+\left(\frac{1}{\alpha^{2}}-1\right)\left|F^{T} n\right|-3\right] \\
\infty & \text { if } \operatorname{det} F=\alpha \\
\infty & \text { else. }\end{cases}
\end{aligned}
$$

Here

$$
w_{0}=\frac{U_{0} k_{0}}{\left|U_{0} k_{0}\right|},
$$

and

$$
N^{(F)}=N_{1}^{\left(F U_{0}^{-1}\right)}=\left\{n \in S^{2}: n \cdot \frac{F^{-T} w_{0}}{\left|F^{-T} w_{0}\right|}=\cos \theta\right\} .
$$

Notice that $\left|U_{0} k_{0}\right|=1 /\left|U_{0}^{-1} w_{0}\right|$. Further, the director at cross-linking is the fixed vector $n_{0} \in N_{1}^{(\mathrm{Id})}=N^{\left(U_{0}\right)}$.

It remains to show that the function $W_{\mathrm{C}}$ defined in (A.1) is nonnegative, its minimum is zero, and is attained by matrices in the set defined in (2.2), namely,

$$
K_{\mathrm{C}}=S O(3)\left\{\operatorname{Id}+(\alpha-1) n \otimes n: n \cdot w_{0}=\beta\right\} .
$$

To see this, we observe that the square bracket in (A.1) satisfies

$$
|F|^{2}+\left(\frac{1}{\alpha^{2}}-1\right)\left|F^{T} n\right|-3 \geq \lambda_{1}^{2}(F)+\lambda_{2}^{2}(F)+\frac{1}{\alpha^{2}} \lambda_{3}^{2}(F)-3,
$$

where $\lambda_{i}$ are the ordered singular values of $F$, with equality holding if $\left|F^{T} n\right|=$ $\lambda_{3}(F)$. Since $\lambda_{1} \lambda_{2} \lambda_{3}=\operatorname{det} F=\alpha$, the right-hand side of (A.3) is minimized when $\lambda_{1}=\lambda_{2}=\lambda_{3} / \alpha=1$, its minimum is zero, and the minimizers are the matrices of the form

$$
F=Q U_{m}
$$

where $m \in S^{2}$, and $Q \in S O(3)$. The left-hand side of (A.3), in turn, is minimized when additionally to the said conditions, $n$ can be chosen so that (A.3) becomes an equality. This is equivalent to provided that $n=Q m \in$ $N^{(F)}$, i.e.,

$$
Q m \cdot \frac{F^{-T} w_{0}}{\left|F^{-T} w_{0}\right|}=\cos \theta .
$$

The second term in (A.1) is also nonnegative, hence $W_{\mathrm{C}} \geq 0$. We now check that the minimum of $W_{\mathrm{C}}$ is zero, and that it is attained by the matrices 
of the form given in (A.2). To do this, it suffices to show that

$$
K_{\mathrm{C}}=\left\{F: F=Q U_{m},\left|F^{-T} w_{0}\right|=\left|U_{0}^{-1} w_{0}\right|, \quad Q m \cdot \frac{F^{-T} w_{0}}{\left|F^{-T} w_{0}\right|}=\cos \theta\right\},
$$

where as above $m \in S^{2}, Q \in S O(3)$. Equivalently, we have to show that

$$
K_{\mathrm{C}}=\left\{F: F=Q U_{m},\left|U_{m}^{-1} w_{0}\right|=\left|U_{0}^{-1} w_{0}\right|, U_{m}^{-1} m \cdot w_{0}=\left|U_{0}^{-1} w_{0}\right| \cos \theta\right\},
$$

The second condition is of the form $m \cdot w_{0}=\beta$, with

$$
\beta=\left|U_{0}^{-1} w_{0}\right| \alpha \cos \theta .
$$

In particular, it is fulfilled by $m=n_{0}$. Since $\left|U_{m}^{-1} w_{0}\right|$ depends only on the scalar product $m \cdot w_{0}$, it is constant on the set $K_{\mathrm{C}}$, hence equality is proven.

\section{References}

[1] J. M. Adams and M. Warner, The elasticity of smectic-A elastomers, Phys. Rev. E 71 (2005), 021708.1-15.

[2] J. M. Adams and M. Warner, Soft elasticity in smectic elastomers, Phys. Rev. E 72 (2005), 011703.1-8.

[3] J. M. Ball and R. D. James, Fine phase mixtures as minimizers of the energy, Arch. Ration. Mech. Analysis 100 (1987), 13-52.

[4] _ Proposed experimental tests of a theory of fine microstructure and the two-well problem, Phil. Trans. R. Soc. Lond. A 338 (1992), $389-450$.

[5] K. Bhattacharya, Microstructure of martensite: Why it forms and how it gives rise to the shape-memory effect, Oxford University Press, 2003.

[6] S. Conti, A. DeSimone, and G. Dolzmann, Semi-soft elasticity and director reorientation in stretched sheets of nematic elastomers, Phys. Rev. E 66 (2002), 061710.1-8.

[7] _ Soft elastic response of stretched sheets of nematic elastomers: a numerical study, J. Mech. Phys. Solids 50 (2002), 1431-1451.

[8] S. Conti, A. DeSimone, G. Dolzmann, S. Müller, and F . Otto, Multiscale modeling of materials - the role of analysis, Trends in Nonlinear Analysis (Heidelberg) (M. Kirkilionis, S. Krömker, R. Rannacher, and F. Tomi, eds.), Springer, 2002, pp. 375-408. 
[9] B. Dacorogna, Direct methods in the calculus of variations, SpringerVerlag, New York, 1989.

[10] B. Dacorogna and P. Marcellini, Implicit partial differential equations, Progress in Nonlinear Differential Equations and their Applications, 37, Birkhäuser, 1999.

[11] A. DeSimone and G. Dolzmann, Macroscopic response of nematic elastomers via relaxation of a class of $S O(3)$-invariant energies, Arch. Rat. Mech. Anal. 161 (2002), 181-204.

[12] G. Dolzmann, Variational methods for crystalline microstructure - analysis and computation, Lecture Notes in Mathematics, no. 1803, SpringerVerlag, 2003.

[13] G. Dolzmann and B. Kirchheim, Liquid-like behavior of shape memory alloys, C. R. Math. Acad. Sci. Paris 336 (2003), 441-446.

[14] K. Hiraoka and H. Finkelmann, Uniform alignment of chiral smectic $C$ elastomers induced by mechanical shear field, Macromol. Rapid Commun. 22 (2001), 456-460.

[15] K. Hiraoka, W. Sagano, T. Nose, and N. Finkelmann, Biaxial shape memory effect exhibited by monodomain chiral smectic $C$ elastomers, Macromolecules 38 (2005), 7352-7357.

[16] B. Kirchheim, Deformations with finitely many gradients and stability of quasiconvex hulls, C. R. Acad. Sci. Paris Sér. I Math. 332 (2001), 289-294.

[17] S. Müller, Variational models for microstructure and phase transitions, in: Calculus of variations and geometric evolution problems (F. Bethuel et al., eds.), Springer Lecture Notes in Math. 1713, Springer-Verlag, 1999, pp. 85-210.

[18] S. Müller and V. Šverák, Convex integration with constraints and applications to phase transitions and partial differential equations, J. Eur. Math. Soc. (JEMS) 1 (1999), 393-442.

[19] E. Nishikawa and H. Finkelmann, Smectic-A liquid single crystal elastomers - strain induced break-down of smectic layers, Macromol. Chem. Phys. 200 (1999), 312-322. 
[20] E. Nishikawa, H. Finkelmann, and H. R. Brand, Smectic-A liquid single crystal elastomers showing macroscopic in-plane fluidity, Macromol. Rap. Comm. 18 (1997), 65-71.

[21] R. Stannarius, R. Köhler, U. Dietrich, M. Löscher, C. Tolksdorf, and R. Zentel, Structure and elastic properties of smectic liquid crystalline elastomer films, Phys. Rev. E 65 (2002), 041707.1-11.

[22] R. Stannarius, R. Köhler, M. Rössler, and R. Zentel, Study of smectic elastomer films under uniaxial stress, Liquid Crystals 31 (2004), 895906.

[23] O. Stenull and T. C. Lubensky, Phase transitions and soft elasticity of smectic elastomers, Phys. Rev. Lett. 94 (2005), 018304.1-4.

[24] V. Šverák, On the problem of two wells, Microstructure and phase transition, IMA Vol. Math. Appl., vol. 54, Springer, New York, 1993, pp. 183189.

[25] M. Warner and E. M. Terentjev, Liquid crystal elastomers, Oxford Univ. Press, 2003. 